\title{
Anomalous left hepatic vein to coronary sinus in a patient with atrial septal defect : Minimally invasive approach ; Technical challenges
}

\author{
Rajesh Rao ${ }^{1}$, Varadraju R ${ }^{2}$, Girish Basappa ${ }^{2}$, and Naveen Sing $^{2}$ \\ ${ }^{1}$ Sri Jayadeva Institute of Cardiology \\ ${ }^{2}$ Sri Jayadeva Institute of Cardiovascular Sciences and Research
}

July 11, 2021

\begin{abstract}
Left hepatic vein draining into coronary sinus is a rare systemic vascular anomaly. Its presence is significant when it is associated with other cardiac lesions requiring surgery. We report technical challenges in a case of persistent left superior vena cava and left hepatic vein draining into coronary sinus in an adult with ostium secundum atrial septal defect, which was repaired through minimally invasive approach. Main technical challenge in this case was to achieve adequate venous drainage, which was achieved by vacuum assistance and by manipulating the position of femoral venous cannula. We approached through right anterolateral thoracotomy, adequate venous drainage was achieved without cannulating left hepatic vein or left superior vena cav.
\end{abstract}

\section{Anomalous Left Hepatic Vein to Coronary Sinus In A Patient With Atrial Septal Defect : Minimally Invasive Approach ; Technical challenges.}

Rajesh K Rao ${ }^{* 1} \mathrm{MCh}$, Varadaraju R ${ }^{1} \mathrm{MCh}$, Girish B ${ }^{1} \mathrm{MCh}$, Naveen Singh ${ }^{2}$ DM

* Corresponding author, Professor, Department of Cardiac Surgery

Sri Jayadeva Institute of Cardiovascular Sciences \& Research, Jayanagar $9^{\text {th }}$ Block, B.G.Road, Bengaluru- 560069, Karnataka, India.

Mobile : +919886964031

E-mail : rajesh.mk5207@gmail.com

1 Department of Cardiac Surgery, Sri Jayadeva Institute of

Cardiovascular Sciences \& Research

2 Department of Cardiac Anesthesia, Sri Jayadeva Institute of

Cardiovascular Sciences \& Research

Rajesh K Rao . ORCID ID 0000-0001-8117-2776

Varadraju R. e-mail; jphm414@gmail.com ORCID ID 0000-0003-0568 -3990

Girish B. e-mail; girishb1009@gmail.com ORCID ID 0000-0003-1923-7799

Naveen Singh. e-mail; navsing5207@gmail.com ORCID ID 0000-0003-2700-1540 
Conflict of interest : None declared

ABSTRACT : Left hepatic vein draining into coronary sinus is a rare systemic vascular anomaly. Its presence is significant when it is associated with other cardiac lesions requiring surgery. We report technical challenges in a case of persistent left superior vena cava and left hepatic vein draining into coronary sinus in an adult with ostium secundum atrial septal defect, which was repaired through minimally invasive approach. Main technical challenge in this case was to achieve adequate venous drainage, which was achieved by vacuum assistance and by manipulating position of femoral venous cannula. We approached through right anterolateral thoracotomy, adequate venous drainage was achieved without cannulating left hepatic vein or left superior vena cava.

Clinical trial registration : $\mathrm{N} / \mathrm{A}$

International Review Board approval : N/A

Informed consent was obtained from the patient

INTRODUCTION : Left hepatic vein (LHV) draining into coronary sinus(CS) is a rare vascular anomaly ${ }^{[1]}$ - It has no hemodynamic or clinical significance, unless it is associated with other cardiac anomalies requiring surgical correction. Unlike median sternotomy, access to LHV is difficult in minimally invasive surgery due to limited exposure and its position with respect to inferior vena cava (IVC), as seen by the operating surgeon. Main challenges in such cases are looping IVC and achieving adequate venous drainage during cardio pulmonary bypass (CPB).

CASE REPORT : A 28 year old female with diagnosis of congenital heart disease ,ostium secundum atrial septal defect (ASD) with persistent left superior vena cava (PLSVC) was referred for surgical correction. She had a massively dilated CS, which is not usually seen in a case of PLSVC. Magnetic resonance angiography (MRA) was done for further evaluation, which showed anomalous LHV draining into CS (Figure 1)

We approached through right anterolateral thoracotomy (RALT), with total peripheral cannulation. After induction with single lumen endotracheal tube transesophageal echocardiogram (TEE) probe was placed. After partial heparinization (1- $1.5 \mathrm{mg} \backslash \mathrm{kg}$ ) right internal jugular vein was cannulated percutaneously using 16 Fr Edwards cannula. A $4-5 \mathrm{~cm}$ sub mammary incision was made and right pleural cavity was entered through $4^{\text {th }}$ intercostal space. Pericardiotomy was done $3 \mathrm{~cm}$ anterior to the phrenic nerve and stay sutures are placed. Right femoral vessels are exposed. After full heparinization, femoral artery and vein are cannulated with $19 \mathrm{Fr} \& 21 \mathrm{Fr}$ Biomedicus cannulae respectively by Seldinger technique. Instead of placing femoral venous cannula tip at the IVC right atrial junction, it was placed in the right atrium to accommodate venous return from CS. Superior vena cava was looped. We did not attempt looping IVC or LHV as the space between them was less and also LHV was behind IVC(as seen by the operating surgeon).

CPB was established with the help of vacuum. After applying aortic cross clamp (Chittwood clamp) ,cold blood cardioplegia was administered through root. Just before atriotomy femoral venous cannula was pulled back to IVC right atrial junction.ASD was closed using autologous pericardium. During intra cardiac repair venous return from LHV and PLSVC was managed by keeping a cardiotomy suction in the CS. Femoral venous cannula was pushed back into right atrium while taking last few stiches over the atrium. Uneventfully weaned off from the CPB, rest of the intra operative and post operative period was uneventful.

DISCUSSION : LHV to CS is rare congenital vascular anomaly ${ }^{[1]}$.It is due to persistent connection between left vitelline vein system and left horn of sinus venosus. Commonly associated anomalies with this condition are PLSVC, subaortic stenosis, anomalous pulmonary venous drainage, atrial and ventricular septal defects, duplication of superior vena cava or $\operatorname{IVC}^{[2,3]}$. It has no clinical or hemodynamic significance unless it is associated with other cardiac anomalies requiring surgical correction. If not detected preoperatively, this anomaly is found during IVC cannulation or looping ${ }^{[4]}$. Preoperative diagnosis helps to plan the proposed surgery properly.

PLSVC can be easily diagnosed by transthoracic echocardiogram, with or without saline agitation test. 
However LHV to CS is missed easily due to limited spatial resolution and limited window with narrow fields of view. Computed tomography angiography and MRA are more accurate and non invasive imaging modalities which provide detailed anatomic information, which helps in planning surgery ${ }^{[5]}$.

During surgery blood flow from LHV can be controlled by a torniquet or by direct cannulation ${ }^{[3]}$. Care must be taken to avoid traction on LHV, as it may cause hemodynamic compromise or arrhythmias. However access to LHV becomes difficult in minimally invasive surgeries due to limited exposure and its position with respect to IVC (lies behind IVC, as seen by the operating surgeon)

Two main challenges in such cases approached through minimal access approach are 1)looping IVC 2) achieving adequate venous drainage on CPB. We did not loop IVC as the gap between IVC and LHV was less and access was also difficult. Venous drainage was assisted by vacuum ${ }^{[6]}$.Adequate venous drainage was achieved by manipulating the position of femoral venous cannula and by choosing one size larger cannula. At the initiation of CPB femoral venous cannula was placed in the right atrium. Just before atriotomy it was pulled back to its regular position(IVC right atrial junction), again pushed back into right atrium while taking last few stiches over the right atrium. During intra cardiac repair venous drainage was achieved by placing cardiotomy suction in the CS.

Another method to achieve adequate venous drainage is by triple venous cannulation. Along with right internal jugular and femoral vein ,right atrial appendage is cannulated. Right atrial cannula is clamped just before atriotomy and unclamped while taking last few stiches over right atrium. Other options to achieve adequate venous drainage are, kinetic assisted drainage or use of new generation or virtually wallless cannulas ${ }^{[6,7]}$.

CONCLUSION : LHV draining into CS even though rare, it is significant when associated with other cardiac anomalies requiring surgical correction.Pre operative diagnosis helps to plan the surgery properly. During surgery main challenges are IVC looping and achieving adequate venous drainage on CPB. This becomes even more challenging in minimally invasive surgery due to limited exposure and poor access to LHV and PLSVC. Care must be taken while looping IVC or avoid looping and use vacuum to assist drainage. Adequate venous drainage can be achieved by choosing one size larger femoral venous cannula and by manipulating its position on CPB. Another option is triple venous cannulation, by additionally cannulating right atrial appendage with a smaller cannula and clamping it just before atriotomy and unclamping it while taking last few stiches over right atrium.

\section{References:}

1. Varun C, Ozker E, Gumus B, Turkoz R. Anomalous hepatic vein draining into coronary sinus. Pediatr Cardiol 2011;32(8):1256- 57

2. Buehler M, Abdullah A, Lewis TJ. Left hepatic vein and persistent left superior vena cava drainage into the coronary sinus with subaortic valve stenosis. Int J Angiol 2011;20(4):243-46

3. Karolczak M A, Madry W, Zacharska-Kokot E.Anomalous connection of left hepatic vein to coronary sinus in a child with PAPVD; Surgical significance and diagnostic difficulties. Kardiochir Torakochirurgia Pol 2016;13(1):49- 51

4. Morshuis WG, de Lind Wijingaarden RA, Kik C, Bogers AJ. Drainage of left hepatic vein into the coronary sinus, a rare intraoperative finding. J Card Surg 2015;30(11):817-18

5. Bhatti S, Hakeem A, Ahmed U, Malik M, Kosolchareon P, Chang S M.Persistent left superior vena cava with anomalous left hepatic vein drainage into the right atrium : role of imaging and clinical relevance. Med 2007;12(4):319- 24

6. Corno .A .F. Systemic venous drainage :can we help Newton? Eur J Cardiothorac Surg 2007;31:1044-51

7. Ludwig Karl von Segesser, Denis Berdajs, Saad Abdel Sayed, Piergiorgio Tozzi, Enrico Ferrari, Francesco Maisano. New virtually wal-less cannulas designed for augmented venous drainage in minimally invasive cardiac surgery. Innovations $2016 ; 11(4): 278-81$

Figure Legend : 
Figure 1: Coronal contrast enhanced MRA image showing LHV (red arrow), PLSVC (blue arrow) draining into CS and IVC (black arrow) draining into right atrium.

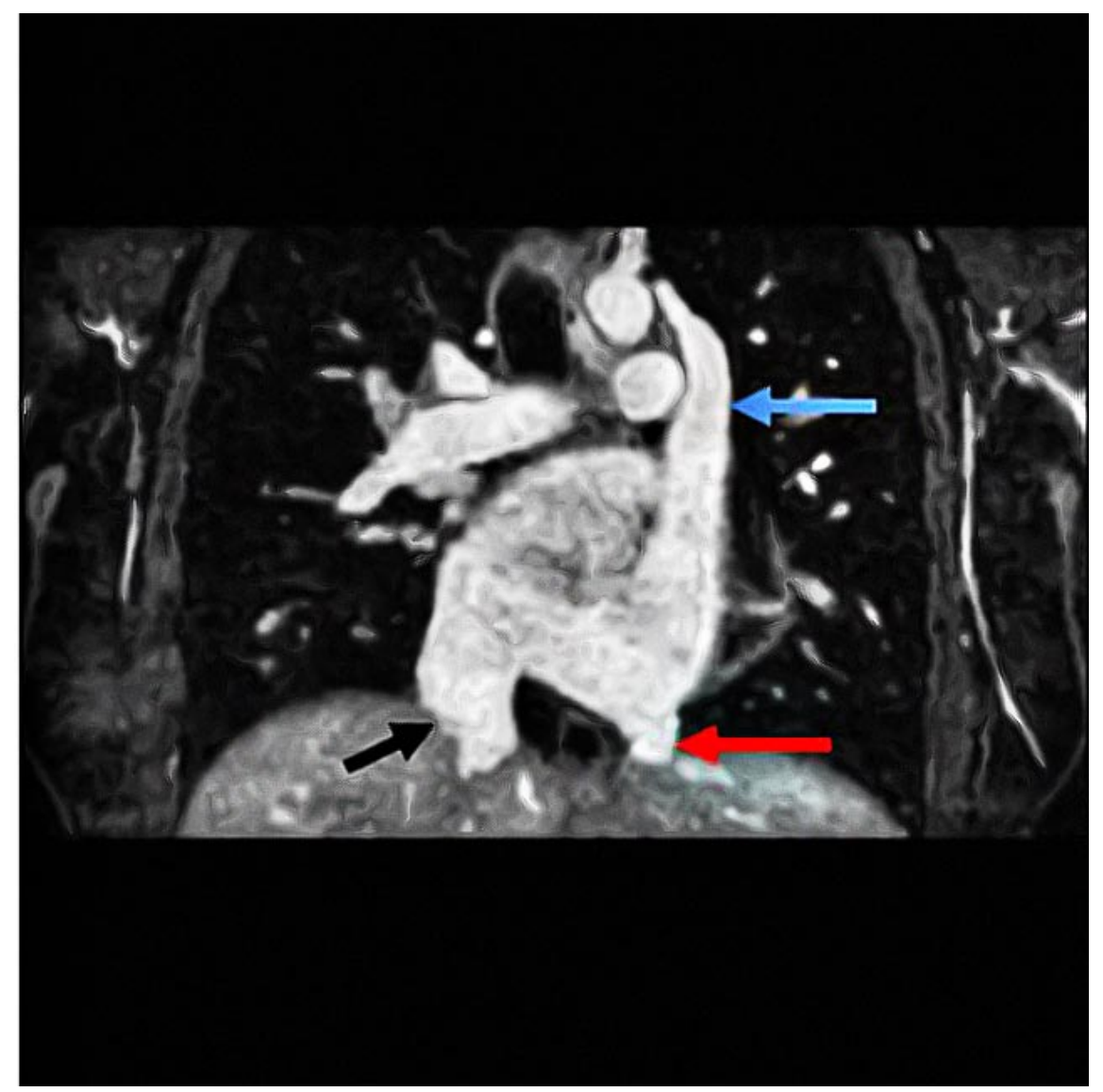

